\title{
Study on the Method of CGF Intelligent Decision-making
}

\author{
Xiaoming Du,Shuchao Tian, Xuefeng Xu,Gu Ping \\ Command and Management Department \\ Mechanical Engineering College \\ Shijiazhuang,China
}

512929156@qq.com

\begin{abstract}
The intelligent decision-making method applied to the CGF behavior modeling is studied more and more. In this paper, we introduce the intelligent decision CGF definition, method, and the characteristics. It analyzes the general process of CGF intelligent decision, and constructs the CGF intelligent decision model based on rule reasoning. The key research of CGF intelligent decision model decision is the knowledge representation, and it puts forward the definition of the sensitive situation and key node to make reasoning process of CGF intelligent decision more concise and convenient. The CGF intelligent decision-making method of reasoning mechanism based on rule reasoning is close to human thinking mode, and the logic structure is easy to grasp and realize.
\end{abstract}

Keywords-computer generated forces; decisionmaking;rule

\section{INTRODUCTION}

Computer Generated Forces is the force that is generated by the computer in the simulation environment, it is the simulation entity ${ }^{[1]}$. Earlier CGF does not have the intelligent decision ability, decision-making behavior is accomplished by the persons involved in the simulation. With the development of modeling theory, artificial intelligence, computer technology, CGF has gradually developed in the independent decision-making ability.

At present, in the field of military simulation, intelligent decision-making method of CGF mainly has the method based on rule reasoning, the method based on the artificial neural network, the method based on genetic algorithm, the method based on the case reasoning. The rule reasoning method is very closed to the mode of human thinking, and the logic structure is easy to grasp. This paper puts forwards a method of rule reasoning, uses the production rules to describe the CGF decision, and implements the CGF decision process through the reasoning of the decision rules.

\section{THE CGF INTELLIGENT DECISION MODEL}

\section{A. The meanings and contents of the decision}

The decision is the thinking process that the people search for the satisfactory solution or optimal solution ${ }^{[2]}$. It generally includes three aspects: the first is the decision of description and representation; the second is the candidate decision scheme to solve the decision problem, and choose the satisfactory scheme or optimal solution according to the principle, method and standard of evaluation and decision scheme; the third is that when we get the satisfied and optimal solution, ending the decision-making process, or not, modify the program, and take the modified scheme into the next round of candidate decision scheme.
CGF intelligent decision refers to the decision that the CGF assistant decision-making in combat simulation system in process when it is similar to the decision ability of people. In the CGF system, intelligent decision is realized by programming. Through the program, we give CGF some intelligent decision ability to deal with various complex situation and complex problems without intervention $^{[3]}$. CGF intelligent decision has the factors like the complexity of simulation object, the adaptability of the method, the results of confirmatory, multifarious of knowledge and the real-time of decision, and so on, which makes the development of the CGF intelligent level is limited.

\section{B. The general process of CGF decision}

CGF intelligent decision generally contains two levels: individual level and group level ${ }^{[4]}$, and the intelligent decision-making group level is based on the individual level. This paper mainly studies the CGF intelligent decision of individual level. For the intelligent decision of CGF, it is critical to simulate the processes of the commanders to collect and crew information, decisionmaking reasoning, coordination control through computer program. Generally, a complete decision process includes the collecting information, organizing information, decision-making reasoning, mission planning, outputting the decision, and so on. The basic process is shown in figure 1 .

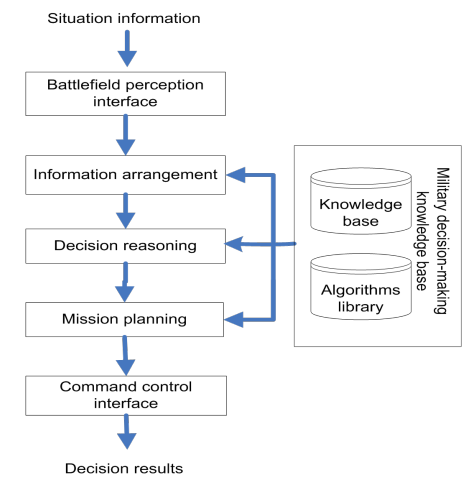

Figure 1. The general intelligent decision process

When CGF makes the decision, it is need to observe and obtain the useful information through the battlefield perception interface from the battlefield, the information processing module interprets it, update, classification, and then sent to the decision-making reasoning module. Decision-making reasoning module based on the battlefield situation information, according to the military decision-making knowledge rule base, identifying and planning decision scheme, through the command control interface will decision results are transmitted to the 
physical model, the driving physics model to complete the corresponding action.

\section{The CGF intelligent decision model}

CGF intelligent decision process can be considered that it is a process of searching for military decision knowledge and finding out the corresponding measures according to the situation information. CGF intelligent decision-making method based on rule reasoning is describing the military decision-making knowledge with rules, and realizing the decision reasoning process based on the rules reasoning. According to the general process of CGF intelligent decision, the decision model is shown in figure $2^{[5]}$.

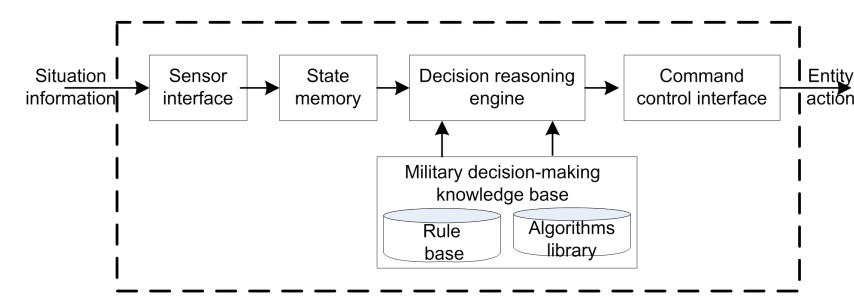

Figure 2. CGF intelligent decision model based on rule reasoning.

As it is shown in Figure 2, the CGF decision model gets battlefield information and stores in the memory through the sensor interface; then the state memory analyzes, classifies, storages the information, and provides decision reasoning engine ; the military decision-making knowledge base includes rules library and algorithms library. The rule description of rules library is the action when meeting a certain situation, the algorithms description of algorithm library is a mathematical operation and decision-making operations used in the reasoning process; with the state information in the state memory, the decision reasoning engine uses the decision algorithm of rules knowledge and algorithms library in the rules base to complete reasoning process and generate decision results; the decision results outputs through command decision interface, and drives the physical model to complete the corresponding entity action.

\section{CGF INTELLIGENT DECISION METHOD BASED ON RULE REASONING}

\section{A. The construction of rule reasoning system .}

Rule reasoning system knowledge is represented by the production rule, including production rule base, the interpreter and the global database, its basic structure is shown in figure 3.

1) The production rule base.Production rule base contains a series of rules, form as follows:

IF ( condition ) ... THEN ( operation or conclusion )

In production rule representation, the IF part is called the conditions or the preceding part, referred to the conditions that the corresponding rules should meet; the THEN part is called the conclusion, operation or consequent, referred to the results that it shall be executed under certain conditions. In the rule reasoning system, if meet a rule condition, the rule reasoning system will control the operation part of the execution rules. The rules in the database use BNF paradigm for storage in the form shown below:

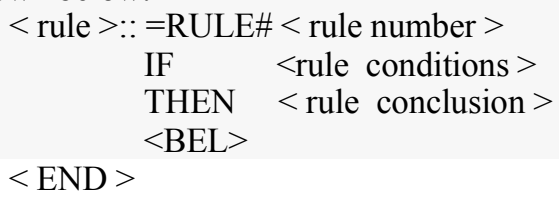

$<$ END $>$

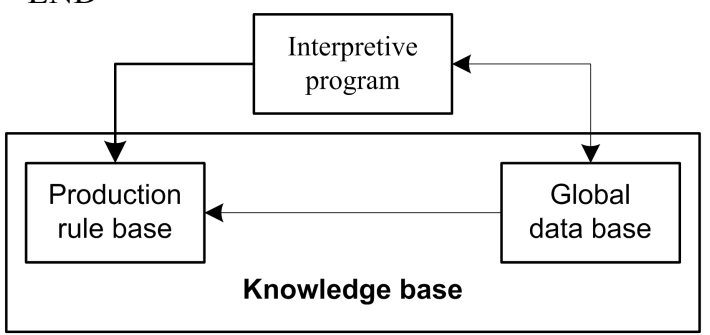

Figure 3. The rule of inference system.

2) The global data base. The global database refers to a group of truth about the world. The preceding part of the production rules refers to the condition in the global database that enabling a rule must comply with, performing it will cause changes in the global database.

For example, when loading ammunition, the ammunition support crew must meet the situation like suitable types, sufficient quantity, no thunderstorm weather, no enemy threat, and enough support entity transport ability. If we enable the rules, the entities begin to load ammunition; and ammunition loading is completed, the corresponding number in ammunition depot will reduce, then the support entities start moving.

3) Interpreter. The interpreter tells us that which rules we should obey, including matching, conflict resolution and operation. Matching refers to that we compared the current global database with rule conditions part, if match, then enable the rules, or not, not enable. Conflict resolution refers to that which rules we should choose when there are several rules enabled. Operation is operating part when we implement the rules. Once operation is performed, the global database will be modified.

In summary, rule reasoning is to match the state description of global database and the conditions of production rule base, then judge matching results by the interpreter, determine the rule whether it is enabled or not, and changes of the global database after enabling rules.

\section{$B$. The representation of decision rules knowledge}

In the CGF intelligent decision based on the rules reasoning, we use the description of decision rules to describe the action under a certain condition. The decision rule is derived from the military decision knowledge of actual command decision-making persons, including combat doctrine, combat principle, expert experience, and so on ${ }^{[6]}$. Generally, we use production rules (IF ... THEN) form. In the CGF system, the state information of decision reasoning is composed of a large number of state factors, these situational factors change any time, but not all of changes will cause the change of the entity and action. When some sensitive situation exceeds a certain value, it will cause the changes of the entity action and movement. We call it the sensitive statement, and the value which causes the change of entity state is called key node. The reasoning process of decision reasoning system can be 
seen as a change of the entity action or movement when searching the sensitive situation information continuously and a sensitive statement achieve to the key node.

We suppose the ammunition supply of tactical ammunition crew under the battlefield conditions for an example. The influence factors on ammunition supply mainly contain: the task, the situation of enemy, the situation of our forces, the situation of friendly forces, the terrain, the climate, electromagnetic, etc. And the state factors influence more on the ammunition supply is the task, the situation of enemy, the terrain and climate, they are called as sensitive situation.

Climatic factors mainly describe the influence on the moving speed of CGF entity. We set the climate factors to describe the different influence on speed. Obviously, the sunny and cloudy days have different influences on the speed. The influence on the moving speed is rain, snow, and fog.We suppose that climatic influence factor is 1 , the corresponding relationships are shown in table 1.

TABle I. Relationship Between Climate and Climatic FaCtors INFLUENCING THE CORRESPONDING

\begin{tabular}{|c|c|c|}
\hline climate & Climatic Factors & Effect Degree \\
\hline fine/rain weather & 1 & Without \\
\hline light rain & 1.5 & Small \\
\hline moderate rain & 2 & Moderately Big \\
\hline downpour rain & 2.5 & Big \\
\hline heavy rain & 3 & Very Big \\
\hline
\end{tabular}

Obviously, when the climate is fine from rain, the climate influence value becomes larger, and it will affect the speed of the CGF entity, which will cause CGF decision changing, so we call the climate influence value as the key node.

\section{Decision method, decision inference system}

The reasoning process can be regarded as a process of continuous searching for sensitive situation information, and reasoning in the key node. In the process of reasoning, we should search for the sensitive state information, determine the changes of the key node of the sensitive situation, if changed, trigger the decision rule (matching current state information and decision rules), after matching, out of the solution and operating, we can get a final decision rule for use. According to output action element of the decision rules, the process is shown in figure 4 .

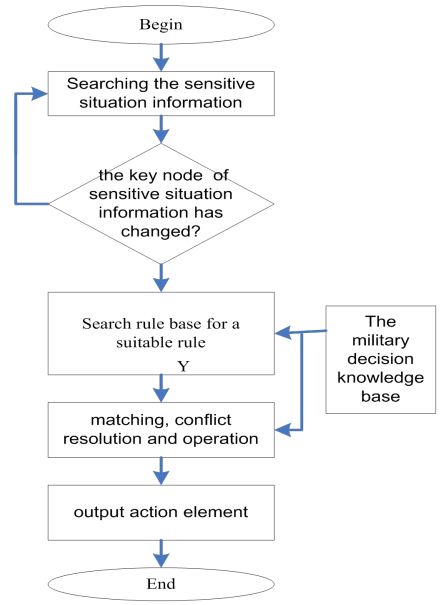

Figure 4. Decision inference flow chart.
In the decision-making process, the key node of sensitive situation information may be changed more than 2 at the same time, and it is related to the conflicting resolution problem. Conflicting resolution strategy can take the method of rules of order, data sorting and nearby ranking. In the generation process of conflict resolution after the decision, the decision result of the decision reasoning will drive the physical action of CGF entity, and the result could not describe all of the elements that the physical action of CGF entity which requires the right algorithm in the algorithm library to calculated the other action elements in the operation process according to the description of rule result. In order to get the final decision result, the decision reasoning based on rules should use the rule reasoning and quantitative calculation to drive the physical action of CGF entity.

\section{CONCLUSION}

It is difficult and important to implement the autodecision of CGF entity in the CGF modeling, and there haven't been any complete theoretical systems. This paper uses the method based on the rule reasoning to implement the intelligent decision-making action of CGF entity, and describe the decision rule according to the military decision knowledge, and implement the decision reasoning process with the decision rule reasoning. This paper introduces the CGF decision process, decision model, the description method of decision rule knowledge and decision reasoning method. The CGF decision method based on rule reasoning is similar to human's thinking mode, the logic structure is easy to master by military person ${ }^{[7]}$, and also to be understood, maintained and realized.

\section{REFERENCES}

[1] R.P.Yang and X.D.Huang,The Intellectualized Decision-makong Methods of Computer Generated Forces and Their Application.Beijing:Publishing House of Electronics Industry,2011.

[2] Y.B.Wu,Q.Jia,W.G.Wang and Y.F.Zhu, "The Research on Decision-Making of the Combat Entity of Naval Tactical Simulation based on Rule Reasoning,"Fire Contuol\&Command Control,vol. 34,no.8,pp.30-33,2009.

[3] QingSui,Ye-ChuanYeo,Khee-YinHow."An Advanced Rule Engine for Computer Generated Forces,"SISO2008 Conference On Behavior Representation in Modeling and Simulation(BRIMS),pp.45-50,2008.

[4] FeiMa-Yao,HongGong-Guang."A Modeling and Simulation Framework for Computer Generate Force,'International Conferenceon Smart Manufacturing Application, pp.9094,2008.

[5] J.Basilico,Z.Benz,K.R.Dixon.”The Cognitive Foundry:A Flexible Platform for Intelligent Agent Modeling,"SISO2008 Conference On Behavior Representation in Modeling and Simulation(BRIMS),pp.08-19,2008.

[6] X.Y.Yang,G.H.Gong,and Y.Tian.”Generalized Optimal Game Theory in Virtual Decision-makings,"Control and Decision Conference,pp.1960-1964,2008.

[7] A.ChewHouMing,K.K.Leng,X.Ke.’Providinga Heuristic Method To Simplify Behavior Authoring,'International Joint Conferenceon INC,IMSandIDC,pp.320-324,2009. 